\title{
COEFFICIENTS IN CERTAIN ASYMPTOTIC FACTORIAL EXPANSIONS
}

T. D. RINEY

1. Introduction. Let $p$ and $q$ denote integers with $0 \leqq p \leqq q$, then it is known from $[2 ; 3]$ and $[7]$ that the function

$$
g(w)=\prod_{i=1}^{p} \Gamma\left(w+\sigma_{i}\right) / \prod_{j=0}^{q} \Gamma\left(w+\rho_{j}\right),
$$

where the $\sigma_{i}$ and $\rho_{j}$ are independent of $w$, admits for large $|w|$ in the sector $|\arg w|<\pi-\epsilon$, an asymptotic expansion of the form

$$
g(w)=\left((2 \pi)^{1 / 2}\right)^{1-\alpha} \alpha^{\alpha w+\beta-1 / 2}\left[\sum_{m=0}^{M} c_{m} / \Gamma(\alpha w+\beta+m)\right.
$$

$$
+O(1 / \Gamma(\alpha w+\beta+M+1))] .
$$

Here, and in the sequel, $M$ denotes a non-negative integer independent of $w, \epsilon$ any positive constant and

$$
c_{0}=1 ; \quad \alpha=q+1-p ; \quad \beta=\sum_{j=0}^{q} \rho_{j}-\sum_{i=1}^{p} \sigma_{i}+(1-\alpha) / 2 .
$$

Recently two types of linear recurrence relations satisfied by the $c_{m}$ have been found. The first [5] is an inductive formula for $c_{m}$ depending on all the preceding $c_{n}$; the second [4] gives $c_{m}$ in terms of the preceding $q$ coefficients. An alternative method of obtaining a recursion formula of the latter type has been pointed out by E. M. Wright [6].

J. G. van der Corput [8] has shown that the inverse of $g(w)$ possesses the asymptotic development

$$
\begin{aligned}
(g(w))^{-1}=\left((2 \pi)^{1 / 2}\right)^{\alpha-1} \alpha^{-\alpha w-\beta+1 / 2}[ & \sum_{m=0}^{M} \gamma_{m} \Gamma(\alpha w+\beta-m) \\
& +O(\Gamma(\alpha w+\beta-M-1))]
\end{aligned}
$$

in the sector $|\arg w|<\pi-\epsilon$. Moreover, van der Corput has extended

Presented to the Society, August 27, 1958, under the title of Coefficients in certain asymptotic factorial expansions of the second kind; received by the editors August 7, 1958 and, in revised form, November 6, 1958. 
the method of [5] to obtain a linear recursion formula for $\gamma_{m}, \gamma_{0}=1$, depending on all the preceding $\gamma_{n}$. The purpose here is to present a general method which when applied to $g(w)$ and $(g(w))^{-1}$ yields a finite recursion formula of fixed length $q$ for the $c_{m}$ and $\gamma_{m}$ respectively. ${ }^{1}$

2. General factorial expansion. Let us consider the function

$$
F(x)=\coprod_{j=0}^{q} \Gamma\left(x+s_{j}\right) / \Gamma\left(x+r_{j}\right)
$$

where the $s_{j}$ and $r_{j}$ are independent of $x$. From Stirling's formula it is easy to show that there exist constants $A_{m}, A_{0}=1$, such that in the sector $|\arg x| \leqq \pi-\epsilon F(x)=\sum_{m=0}^{M} A_{m} x^{-b-m}+O\left(x^{-b-M-1}\right)$, where

$$
b=\sum_{j=0}^{q}\left(r_{j}-s_{j}\right) .
$$

The proof which is similar to the proofs cited for (1.2) is omitted. We immediately have the following result which will be stated in the form of a theorem.

TheORem 2.1. Let $d_{m}$ and $a$ be given complex numbers, $|\arg a|<\pi / 2$. Then there exist constants $B_{m}, B_{0}=1$, such that

$$
F(x / a)=a^{b} \sum_{m=0}^{M} B_{m} \Gamma\left(x+d_{m}-m\right) / \Gamma\left(x+d_{m}+b\right)+O\left(x^{-b-M-1}\right)
$$

in the sector $|\arg x|<\pi / 2+\epsilon$ provided $\epsilon<\pi / 2-|\arg a|$.

The problem is to determine an efficient method for computing the coefficients $B_{m}$. For this purpose we shall need the general result of the next section.

3. A general theorem. Let $G(x)$ denote a function analytic in some sector $\left|\arg \left(x-x_{0}\right)\right| \leqq \pi / 2+\epsilon,(\epsilon<\pi / 2)$, such that in this sector

$$
G(x)=O\left(x^{-h}\right),
$$

where $h$ denotes a constant. For such a function, we define

$$
\begin{aligned}
& \psi(t)=L\{G\}=(2 \pi i)^{-1} \int_{C} t^{\lambda x} G(x) d x, \quad(1 \leqq t<\infty \text { if } \lambda>0), \\
& (0<t \leqq 1 \text { if } \lambda<0) \text {, }
\end{aligned}
$$

where $\lambda(\neq 0)$ is real and $C$ denotes the path $\left|\arg \left(x-x_{0}\right)\right|=\pi / 2+\epsilon$ orientated in the upward direction; clearly, $\psi(t)$ does not depend on

${ }^{1}$ We had originally demonstrated the result for $\gamma_{m}$. This method of giving the general result was suggested by the referee. 
the choice of $\epsilon$ or $x_{0}$. Further, $\psi(t)$ is infinitely differentiable, in fact

$$
\psi^{(k)}(t)=\left(\frac{d}{d t}\right)^{k} L\{G\}=t^{-k} L\{\lambda x(\lambda x-1) \cdots(\lambda x-k+1) G(x)\}
$$

Finally, we note the following result:

THEOREM 3.1. $G(x)=O\left(x^{-h}\right)$ implies that near $t=1$

$$
\psi^{(k)}(t)=O\left(|t-1|^{h-k-1}\right),
$$

provided $k<\operatorname{Re}(h)-1$.

Proof. In the integral representation of $\psi^{(k)}(t)$ replace the curve $C$ by $\operatorname{Re}(x)=u \geqq u_{0}=\operatorname{Re}\left(x_{0}\right)$ and set $x=u+i v$ to obtain

$$
\begin{aligned}
& \psi^{(k)}(t)=(2 \pi)^{-1} t^{\lambda u-k} \int_{-\infty}^{+\infty} t^{i \lambda v}(\lambda u+i \lambda v)(\lambda u-1+i \lambda v) \ldots \\
&(\lambda u-k+1+i \lambda v) G(u+i v) d v .
\end{aligned}
$$

There exists a constant $K$, independent of $u$ and $v$, such that

$$
\left|\psi^{(k)}(t)\right|<K \int_{-\infty}^{+\infty} t^{\lambda u-k}\left(\left(u^{2}+v^{2}\right)^{1 / 2}\right)^{k-\operatorname{Re} h} d v .
$$

Substitute $v^{2}=w u^{2}$ to write the last relation in the form

$$
\left|\psi^{(k)}(t)\right|<(K / 2) u^{k-\operatorname{Re} h+1} \int_{-\infty}^{+\infty} t^{\lambda u-k}\left((1+w)^{1 / 2}\right)^{k-\operatorname{Re} h} w^{-1 / 2} d w .
$$

Since the integral is convergent, the assertion follows immediately upon setting $u=|t-1|^{-1}$.

4. The function $\phi(t)$. First apply Theorem 3.1 to the order term in (2.3) to find that, near $t=1$,

$$
\begin{aligned}
\left(\frac{d}{d t}\right)^{h} L\left\{F(x / a)-a^{b} \sum_{m=0}^{M} B_{m} \Gamma\left(x+d_{m}-m\right) / \Gamma\left(x+d_{m}+b\right)\right\} & \\
& =O\left(|t-1|^{b+M-k}\right),
\end{aligned}
$$

provided $k<M+\operatorname{Re}(b)$. It now follows upon introducing

$$
\begin{aligned}
& \phi(t)=L\{F(x / a)\}, \\
& (1 \leqq t<\infty \text { if } \lambda>0), \\
& (0<t \leqq 1 \text { if } \lambda<0) \text {, }
\end{aligned}
$$

and using the well-known relation $[1$, p. 261]

$$
\begin{aligned}
L\left\{\Gamma\left(x+d_{m}-m\right) / \Gamma\left(x+d_{m}+b\right)\right\} & \\
& =(\Gamma(m+b))^{-1} t^{-\lambda\left(d_{m}+b-1\right)}\left(t^{\lambda}-1\right)^{m+b-1},
\end{aligned}
$$


that

$$
\begin{aligned}
\phi^{(k)}(t)= & a^{b} \sum_{m=0}^{M} B_{m}(\Gamma(m+b))^{-1}\left(\frac{d}{d t}\right)^{k}\left[t^{-\lambda\left(d_{m}+b-1\right)}\left(t^{\lambda}-1\right)^{m+b-1}\right] \\
& +O\left(|t-1|^{b+M-k}\right)
\end{aligned}
$$

provided $k<M+\operatorname{Re}(b)$.

Now note that by applying the recurrence relation $\Gamma(x+1)=x \Gamma(x)$ to $(2.1)$, we obtain

$$
F(x / a+1) \prod_{j=0}^{q}\left(x+a r_{j}\right)=F(x / a) \prod_{j=0}^{q}\left(x+a s_{j}\right) .
$$

There exist constants $C_{k}$ and $E_{k}(k=0,1, \cdots, q+1)$ such that

$$
\begin{aligned}
& \prod_{j=0}^{q}\left(x+a s_{j}\right)=\sum_{k=0}^{q+1} C_{k} \lambda x(\lambda x-1) \cdots(\lambda x-k+1), \\
& \prod_{j=0}^{q}\left(x+a r_{j}\right)=\sum_{k=0}^{q+1} E_{k}(\lambda x+\lambda a)(\lambda x+\lambda a-1) \cdots \\
& \quad(\lambda x+\lambda a-k+1) .
\end{aligned}
$$

Relations (4.3) and (4.4) are used to prove the assertion which follows.

THEOREM 4.1. The function $\phi(t)$ defined by (4.1) satisfies the linear differential equation

$$
\sum_{k=0}^{q+1}\left(t^{k+\lambda a} C_{k}-t^{k} E_{k}\right) \phi^{(k)}(t)=0,
$$

where

$$
\begin{aligned}
C_{k} & =(k !)^{-1} \prod_{j=0}^{q}\left(a s_{j}+k / \lambda\right)-\sum_{n=0}^{k-1} C_{n} /(k-n) ! \\
E_{k} & =(k !)^{-1} \prod_{j=0}^{q}\left(a r_{j}-a+k / \lambda\right)-\sum_{n=0}^{k-1} E_{n} /(k-n) !
\end{aligned}
$$

In particular

$$
\begin{aligned}
C_{q+1}=\lambda^{-q-1} ; & C_{q}=\lambda^{-q}\left[a \sum_{j=0}^{q} s_{j}+q(q+1)(2 \lambda)^{-1}\right] \\
E_{q+1}=\lambda^{-q-1} ; & E_{q}=\lambda^{-q}\left[a \sum_{j=0}^{q} r_{j}+(q-2 a \lambda)(q+1)(2 \lambda)^{-1}\right] .
\end{aligned}
$$

Proof. From (3.3) and (4.1) we may write 


$$
\begin{aligned}
\sum_{k=0}^{q+1} E_{k} t^{k} \phi^{(k)}(t) & =L\left\{F(x / a) \sum_{k=0}^{q+1} E_{k} \lambda x(\lambda x-1) \cdots(\lambda x-k+1)\right\} \\
& =t^{\lambda a} L\left\{F(x / a+1) \sum_{k=0}^{q+1} E_{k}(\lambda x+\lambda a)(\lambda x+\lambda a-1)\right. \\
\cdots(\lambda x+\lambda a-k+1)\} & \cdots(\lambda x-k+1)\} \\
= & t^{\lambda a} L\left\{F(x / a) \sum_{k=0}^{q+1} C_{k} \lambda x(\lambda x-1) \cdots(1),\right. \\
& =t^{\lambda a} \sum_{k=0}^{q+1} C_{k} t^{k} \phi^{(k)}(t),
\end{aligned}
$$

which proves assertion (4.5). Equations (4.6) are obtained immediately upon substituting the appropriate values of $x$ into the identities (4.4). Relations (4.7) are obtained by comparing the coefficients of $x^{q+1}$ and $x^{q}$ in the identities (4.4).

5. Recursion formulae. A general technique for obtaining the constants $B_{m}$ in (2.3) is to substitute the asymptotic expansions (4.2) in (4.5), expand all the resulting terms about $t=1$, and equate the coefficients of like powers. This procedure will first be applied to find a finite recursion formula for the constants $\gamma_{m}$ which occur in (1.4).

The method requires that we write (1.4) in the form of (2.3) with $b>q+1$. We choose $b=q+3$ to be consistent with the notation of [4]. In (1.4) set $x=\alpha w+\beta+q+2$ and use the identity

$$
\left((2 \pi)^{1 / 2}\right)^{\alpha-1} \Gamma(x+1)=\alpha^{x+1 / 2} \prod_{\mu=1}^{\alpha} \Gamma(x / 2+\mu / 2)
$$

to find

$$
F_{1}(x / \alpha)=\alpha^{q+3} \sum_{m=0}^{M} \gamma_{m} \Gamma(x-q-2-m) / \Gamma(x+1)+O\left(x^{-q-4-M}\right)
$$

where

$$
\begin{aligned}
F_{1}(x)=\prod_{j=0}^{q} \Gamma\left(x+\rho_{j}-\alpha^{-1}(\beta+q+2)\right) \\
\quad \cdot\left(\prod_{i=1}^{p} \Gamma\left(x+\sigma_{i}-\alpha^{-1}(\beta+q+2)\right) \prod_{\mu=1}^{\alpha} \Gamma\left(x+\alpha^{-1} \mu\right)\right)^{-1} .
\end{aligned}
$$

Equation (5.1) is in the form of (2.3) with 


$$
\begin{aligned}
a & =\alpha ; \quad b=q+3 ; & d_{m}=-q-2(m=0,1, \cdots), \\
s_{j} & =\rho_{j}-\alpha^{-1}(\beta+q+2) & (j=0,1, \cdots q), \\
r_{j} & =\sigma_{j+1}-\alpha^{-1}(\beta+q+2) & (j=0,1, \cdots, p-1), \\
& =\alpha^{-1}(j-p+1) & (j=p, \cdots, q) .
\end{aligned}
$$

If now we choose $\lambda=1$ in (4.2) we have simply

$$
\phi^{(k)}(t) \sim \alpha^{q+3} \sum_{m=0}^{\infty} \frac{\gamma_{m}(t-1)^{m+q+2-k}}{\Gamma(m+q+3-k)} \quad(1 \leqq t<\infty) .
$$

Substitution of (5.4) into (4.5) yields the desired recurrence relation for the constants $\gamma_{m}$.

TheOREM 5.1. The coefficients $\gamma_{m}(m=0,1, \cdots)$, occurring in the expansion (1.4), satisfy the following recursion formula of length $q$ :

$$
\begin{aligned}
\gamma_{m}= & (\alpha m)^{-1}(m+2) ! \\
& \cdot\left\{\sum_{i=1}^{q} \sum_{k=q-i}^{q+1} C_{k}\left(\begin{array}{c}
k \\
q-i
\end{array}\right) \gamma_{m-i} /(m+q-i+2-k) !\right. \\
& \left.-\sum_{i=1}^{p-1} \sum_{k=q-i}^{q+1} E_{k}\left(\begin{array}{c}
k-\alpha \\
q-i-\alpha
\end{array}\right) \gamma_{m-i} /(m+q-i+2-k) !\right\},
\end{aligned}
$$

$\left(\gamma_{0}=1\right.$ and $\left.\gamma_{-1}=\gamma_{-2}=\cdots=0\right)$, where

$$
C_{k}=(k !)^{-1} \prod_{j=0}^{q}\left(\alpha \rho_{j}-\beta-q-2+k\right)-\sum_{n=0}^{k-1} C_{n} /(k-n) !
$$

(5.6) $\quad E_{k}=(k !)^{-1} \prod_{j=0}^{p-1}\left(\alpha \sigma_{j+1}-\beta-q-2-\alpha+k\right) \prod_{j=p}^{q}(j-q+k)$

$$
-\sum_{n=0}^{k-1} E_{n} /(k-n) !
$$

In particular,

$$
C_{q+1}=1 ; \quad C_{q}=\alpha \sum_{j=0}^{q} \rho_{j}-(q+1)(\beta+q+2)+q(q+1) / 2,
$$

$$
\begin{aligned}
E_{q+1}=1 ; \quad E_{q}=\alpha \sum_{i=1}^{p} \sigma_{i}-p(\alpha+\beta & +q+2) \\
& +q(q+1) / 2-\alpha(\alpha-1) / 2 .
\end{aligned}
$$

Proof. Equations (5.6) and (5.7) are immediately obtained by the substitution of (5.2) and (5.3) into (4.6) and (4.7). To find the recursion formula (5.5) set $\lambda=1$ and employ the binomial theorem to write (4.5) in the form 


$$
\begin{aligned}
& \sum_{j=0}^{q+1} \sum_{k=j}^{q+1} C_{k}\left(\begin{array}{l}
k \\
j
\end{array}\right)(t-1)^{k-j} \phi^{(k)}(t) \\
&-\sum_{j=\alpha}^{q+1} \sum_{k=j}^{q+1} E_{k}\left(\begin{array}{c}
k-\alpha \\
j-\alpha
\end{array}\right)(t-1)^{k-j} \phi^{(k)}(t)=0 .
\end{aligned}
$$

By (5.4) the coefficient of $t^{m+2}$ in the asymptotic expansion for $(t-1)^{k-j} \phi^{(k)}(t)$ is

$$
\alpha^{q+3} \gamma_{m-q+j}((m+j+2-k) !)^{-1},
$$

so that, for each integer $m$,

$$
\begin{aligned}
\sum_{j=0}^{q+1} \sum_{k=j}^{q+1} C_{k}\left(\begin{array}{c}
k \\
j
\end{array}\right) \gamma_{m-q+j}((m+j+2-k) !)^{-1} & \\
& -\sum_{j=\alpha}^{q+1} \sum_{k=j}^{q+1} E_{k}\left(\begin{array}{c}
k-\alpha \\
j-\alpha
\end{array}\right) \gamma_{m-q+j}((m+j+2-k) !)^{-1}=0,
\end{aligned}
$$

provided we agree that $\gamma_{m}=0$ for $m<0$. From (5.7), the coefficients of $\gamma_{m+1}$ and $\gamma_{m}$ are 0 and $\alpha m /(m+2)$ ! respectively. The above formula therefore reduces to (5.5) upon solving for $\gamma_{m}$ and setting $i=q-j$.

The general method may also be applied to obtain a recursion formula for the constants $c_{m}$ which appear in (1.2). In this case set $x=\alpha w+\beta-q-3$ and write (1.2) as

$$
\text { (5.8) } \quad F_{2}(x / \alpha)=\alpha^{q+3} \sum_{m=0}^{M} c_{m} \Gamma(x) / \Gamma(x+q+3+m)+O\left(x^{-q-4-M}\right),
$$

where

$$
\begin{aligned}
F_{2}(x)= & \prod_{i=1}^{p} \Gamma\left(x+\sigma_{i}-\alpha^{-1}(\beta-q-3)\right) \\
& \cdot \prod_{\mu=1}^{\alpha} \Gamma\left(x+\alpha^{-1}(\mu-1)\right) / \prod_{j=0}^{q} \Gamma\left(x+\rho_{j}-\alpha^{-1}(\beta-q-3)\right) .
\end{aligned}
$$

Now (5.8) is in the form of (2.3) with

$$
\begin{array}{rlrl}
a & =\alpha ; \quad b=q+3 ; & d_{m}=m, \\
s_{j} & =\sigma_{j+1}-\alpha^{-1}(\beta-q-3) & (j=0,1, \cdots, p-1) \\
& =\alpha^{-1}(j-p) & & (j=p, \cdots, q), \\
r_{j} & =\rho_{j}-\alpha^{-1}(\beta-q-3) & & (j=0,1, \cdots, q) .
\end{array}
$$

If we choose $\lambda=-1$, then (4.2) and (4.5) become simply 


$$
\begin{array}{r}
\phi^{(k)}(t) \sim \alpha^{q+3} \sum_{m=0}^{\infty}(-1)^{m+q} C_{m}(t-1)^{m+q+2-k} / \Gamma(m+q+3-k) \\
(0<t \leqq 1)
\end{array}
$$

and

$$
\sum_{k=0}^{q+1}\left(t^{k-\alpha} C_{k}-t^{k} E_{k}\right) \phi^{(k)}(t)=0
$$

respectively. The recursion formula for the $c_{m}$ obtained by substituting (5.11) into (5.12) is precisely the result given in [4].

6. Remark. When Theorem (5.1) is applied to the special case of

$$
(g(w))^{-1}=\Gamma\left(w+\rho_{0}\right) \Gamma\left(w+\rho_{1}\right)
$$

the result is

$$
\gamma_{m}(8 m)^{-1}\left[4\left(\rho_{1}-\rho_{0}\right)^{2}-(2 m-1)^{2}\right] \gamma_{m-1} .
$$

On the other hand, van der Corput's formula [8, Equation 8] gives

$$
\gamma_{1}=(8)^{-1}\left[4\left(\rho_{1}-\rho_{0}\right)^{2}+71\right] \gamma_{0}
$$

for the second coefficient. This discrepancy has been resolved by H. O. Pollak who has computed $\gamma_{1}$ for this special case by substituting Stirling's formula into (6.1) directly. The result is in agreement with (6.2).

\section{BIBLIOGRAPHY}

1. A. Erdelyi et al., Tables of integral transforms, vol. 1, McGraw-Hill Book Company, Inc., 1954.

2. W. B. Ford, The asymptotic developments of functions defined by Maclaurin series, University of Michigan Studies, Scientific Series, vol. 11, 1936.

3. H. K. Hughes, The asymptotic developments of a class of entire functions, Bull. Amer. Math. Soc. vol. 51 (1945) pp. 456-461.

4. T. D. Riney, A finite recursion formula for the coefficients in asymptotic expansions, Trans. Amer. Math. Soc. vol. 88 (1958) pp. 214-226.

5. - On the coefficients in asymptotic factorial expansions, Proc. Amer. Math. Soc. vol. 7 (1956) pp. 245-249.

6. E. M. Wright, $A$ recursion formula for the coefficients in an asymptotic expansion, to be published in Proc. Glasgow Math. Assoc.

7. - The asymptotic expansion of the generalized hypergeometric function, Proc. London Math. Soc. (2) vol. 46 (1940) pp. 389-408.

8. J. G. van der Corput, On the coefficients in certain asymptotic factorial expansions, Akad. Wet. (Amsterdam) Proc. Series A vol. 60 no. 4 (1957) pp. 337-351.

Bell Telephone laboratories, Inc., Allentown, Pa. 